\title{
Changes in pupil size following panretinal retinal photocoagulation: conventional laser vs pattern scan laser (PASCAL)
}

I Yilmaz, I Perente, B Saracoglu, AT Yazici and M Taskapili

\begin{abstract}
Purpose To evaluate and compare the possible changes in pupil size subsequent to panretinal laser photocoagulation (PRP) via conventional laser and pattern scan laser (PASCAL).

Patients and methods Forty eyes of 40 patients with diabetic retinopathy were included. 20 eyes had a PRP via conventional laser and formed Group 1. 20 eyes had a PRP via PASCAL laser and formed Group 2. The participants underwent standard ophthalmologic examination at baseline. Automated infrared pupillometry were performed at baseline and month 1 .

Results The mean pupillary measurements $(\mathrm{mm})$ for Group 1 (in the order photopic, mesopic, and scotopic conditions) were $2.88 \pm 0.34,3.38 \pm 0.40$, and $3.95 \pm 0.38$, and changed to $3.64 \pm 0.42,4.18 \pm 0.42$, and $4.58 \pm 0.48$, respectively. There was significant increase in pupil size at month $1(P<0.001, P<0.001$, and $P<0.00$, respectively). For Group 2, they were $2.90 \pm 0.38,3.43 \pm 0.36$, and $3.90 \pm 0.40$, and changed to $3.18 \pm 0.42,3.74 \pm 0.36$, and $4.10 \pm 0.38$, respectively. There was significant increase in pupil size at month $1(P=0.018, P=0.014$, and $P=0.014$, respectively). The pupil size increased significantly in both groups in all illumination conditions.

Conclusion We have demonstrated via automated infrared pupillary measurements that PRP may significantly increase pupil size whether it is performed with conventional laser or PASCAL laser.
\end{abstract}

Eye (2016) 30, 1359-1364; doi:10.1038/eye.2016.135; published online 8 July 2016

\section{Introduction}

Retinal laser photocoagulation was introduced in the $1950 \mathrm{~s}^{1}$ and still remains the gold standard for the treatment of peripheral retinal ischemic diseases. Some large multicenter trials supported the positive effects of laser treatment in patients with diabetic retinopathy. ${ }^{2-4}$

However, panretinal laser photocoagulation (PRP) treatment is not perfect. The therapeutic destruction of isolated retinal areas with lasers maybe accompanied by the unavoidable destruction of normal adjacent tissues such as ciliary nerves. ${ }^{5,6}$ Some previous studies have revealed that retinal laser treatments can affect pupillary anatomy, potentially due to short and long ciliary nerves damage. ${ }^{7,8}$ Dilated pupils, loss of accommodation, and tonic pupil have been reported in small case series. ${ }^{78}$ In addition, a dilated pupil may cause photophobia due to the excessive amount of light entering the eye. Also, it may increase ocular aberration and cause visual symptoms.

In this study, we used an automated pupillometry device to measure the pupil diameters in scotopic, mesopic, and photopic conditions before and after the PRP via conventional laser and pattern scan laser (PASCAL). As the impact of PRP on the pupil size of patients with proliferative diabetic retinopathy has not been studied, with the present research, we aimed to evaluate possible pupillary changes subsequent to PRP treatments.
Retina Department, Beyoglu Eye Training and Research Hospital, Istanbul, Turkey

Correspondence: I Yilmaz, Beyoglu Eye Training and Research Hospital, Bereketzade Cami Sokak No: 2 Beyoglu, Istanbul 34408, Turkey Tel: +90 532 3455972; Fax: +90212 2450948 . E-mail: ihsanyilmaz.dr@ gmail.com

Received: 24 November 2015

Accepted in revised form:

5 May 2016

Published online:

8 July 2016 


\section{Materials and methods}

\section{Ophthalmic examination}

Each participant received a standard ophthalmologic examination by an experienced physician (IY) involving refraction, visual acuity, slit-lamp biomicroscopy, Goldmann applanation tonometry, ultrasonic pachymetry, axial length measurements (IOL Master, Zeiss, Jena, Germany), fundus flourescein angiography, and optical coherence tomography (Spectralis, Heidelberg Engineering, Heidelberg, Germany). All automated pupillometry measurements were taken via Sirius (Costruzione Strumenti Oftalmici, Scandicci, Italy) by a masked physician (IP) at the planned laser day before the pupil dilatation and after a month from PRP completion.

\section{Eligibility criteria}

Inclusion criteria required that participants had proliferative diabetic retinopathy (neovascularization elsewhere, neovascularization disc, or both proven via fundus flourescein angiography).

Patients were not included in the study if they had an ocular disease other than diabetic retinopathy, systemic disorders other than diabetes, diabetic macular edema, advance proliferative diabetic retinopathy (neovascularization iris, neovascular glaucoma, or retina detachment), a history of intraocular surgery, active lid or adnexal infection, a history of previous retinal laser therapy or intravitreal injection, a history of systemic medications during the last 3 months, a history of ocular medication during the last year, and a history of ocular or head trauma. Also smokers and heavy alcohol drinkers (drinking five or more drinks on the same occasion on each of five or more days in the past 30 days) were excluded.

\section{Study groups}

The population of suitable cases consisted of all consecutive patients who planned to receive PRP between August and September 2015. First 20 consecutive patients underwent PRP via conventional laser (Visulas $532 \mathrm{~s}$, Zeiss, Carl Zeiss AG, Oberkochen, Germany) and formed Group 1. Second 20 consecutive patients underwent PRP via PASCAL (OptiMedica Corp., Santa Clara, CA, USA) and formed Group 2. If both eyes of a patient qualified for study, then the right eye was designated as the study eye for patients with an even birth month number and the left for those with an odd birth month number. The sample size was calculated using SPSS SamplePower software version 3.0 (IBM, Armonk, NY, USA). On the basis of the research design and strategy, sample size was calculated with $90 \%$ power of study. The minimum requirement for each group was 18 patients. This study was performed in Istanbul and conducted according to the principles of the Declaration of Helsinki. All participants' informed consents were obtained.

\section{Laser protocols}

Conventional laser was used for Group 1 and PRP was completed in three or four sessions, which involved placing roughly 1800 burns of $400 \mu \mathrm{m}$ a half-spot size apart.

PASCAL laser was used for Group 2 and PRP was completed in a single session, which involved placing roughly 2000 burns of $400 \mu \mathrm{m}$ (ie, gray-white burns) a spot size apart with power set to be sufficient to produce a burn of only light intensity.

With both laser devices, the procedure was continued to encompass the entire retina, yet spare the central macular and temporal-nasal midperipheral areas, which entail long posterior ciliary nerves covered with laser burns. Topical anesthesia was adequate for all participants.

\section{Automatic infrared pupillometry measurements}

Sirius is a placido-based videokeratoscope with two Scheimpflug cameras, one central and one rotating (Costruzione Strumenti Oftalmici, Scandicci, Italy). The device allows both static and dynamic pupillometry, and uses different illumination levels to measure pupil size in scotopic (0.04 Lux), mesopic (4 Lux), and photopic (40 Lux) conditions. Figure 1 presents an example of the device's printout.

\section{Pain score (PS) measurements}

PS were administered by asking patients to record their subjective impressions of pain using a scale from 0 (lowest) to 10 (highest). The scale was horizontally oriented, measuring $10 \mathrm{~cm}$, and the value for statistical analysis was measured with a rule at the point where the mark was inserted by the patient. For the conventional laser group, PS was measured after $30 \mathrm{~min}$ from the first session completed. For the Pascal group, PS was measured after $30 \mathrm{~min}$ from the only session completed.

\section{Data analysis}

Data were expressed as mean \pm SD. The KolmogorovSmirnov test was applied to assess the normal distribution of data. To compare before-and-after pupillometric measurements in each group, a paired sample $t$-test was performed. To compare pain responses between laser groups, an independent sample $t$-test was 
a Scotopic (0.04 lux):0.64 mm,-0.13 mm,1.82 mm
Mesopic (4 lux):0.17 mm,-0.15 mm,1.32 mm
Photopic (40 lux):0.42 mm,-0.13 mm,1.16 mm
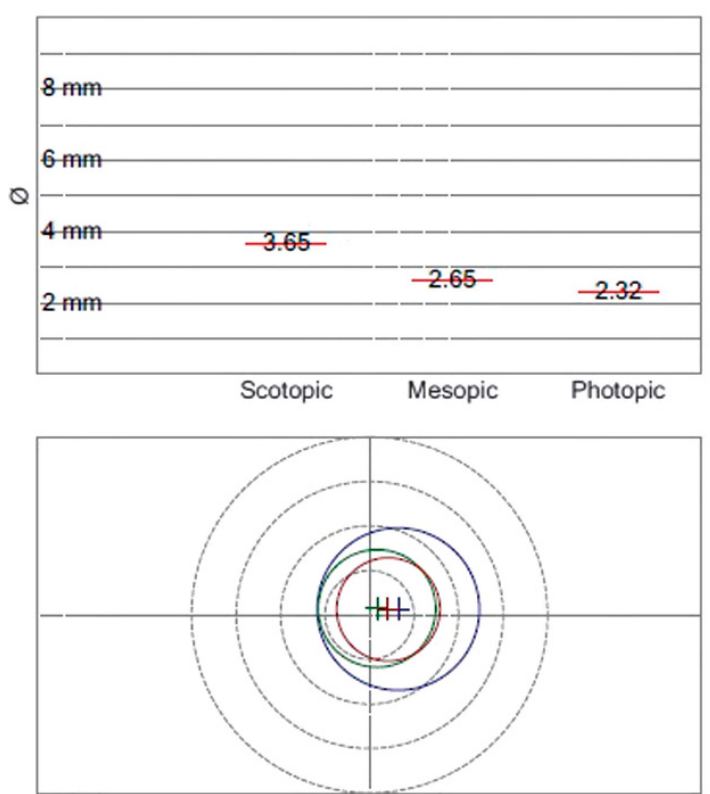

b

Scotopic (0.04 lux): $0.24 \mathrm{~mm}, 0.02 \mathrm{~mm}, 2.25 \mathrm{~mm}$ Mesopic (4 lux):0.27 mm,0.02 mm,2.05 mm Photopic (40 lux):0.23 mm,-0.01 mm, $1.77 \mathrm{~mm}$
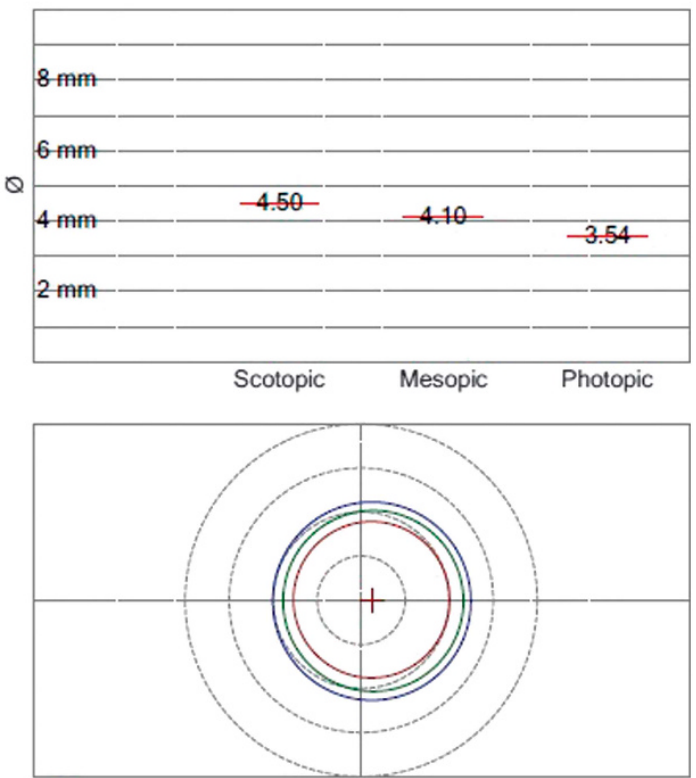

Figure 1 An example of pupillary measurements via Sirius (Costruzione Strumenti Oftalmici); (left eye of the patient from conventional laser group). (a) Before panretinal photocoagulation and (b) 1 month afterward. Original before-and-after printouts are juxtaposed to highlight differences.

performed. The Statistical Package for the Social Sciences version 20 (SPSS, Chicago, IL, USA) was used for data analysis, for which values of $P<0.05$ were considered to be statistically significant.

\section{Results}

\section{Demographic characteristics}

The study sample consisted of 40 eyes of 40 participants (23 females and 17 males), all of whom were Caucasian. Group 1 consisted of 20 eyes of 20 participants ( 11 females and 9 males) and Group 2 consisted of 20 eyes of the other 20 participants (12 females and 8 males).

As shown in Table 1, participants' demographic characteristics such as age, refractive error, visual acuity, axial length, pachymetry, intraocular pressure (IOP), and $\mathrm{HbA1c}$ values were not significantly different between the groups $(P>0.05$ for all); (Table 1$)$.

\section{Laser parameters}

Standard conventional laser photocoagulation is done using single spots with pulse durations of 100-200 ms (Table 2). Multispot laser delivers multiple uniform laser burns simultaneously, by a single foot pedal depression in a variety of patterns. The device saves time and reduces discomfort from the long laser sessions (Table 2).

\section{Pupillary measurements}

The mean pupil sizes of patients in Group 1 (in the order: photopic, mesopic, and scotopic conditions) were $2.88 \pm 0.34,3.38 \pm 0.40$, and $3.95 \pm 0.38 \mathrm{~mm}$ at baseline, and changed to $3.64 \pm 0.42,4.18 \pm 0.42$, and $4.58 \pm 0.48 \mathrm{~mm}$, respectively, after a month from PRP completion (Table 3).

The mean pupil size of patients in Group 2 were $2.90 \pm 0.38,3.43 \pm 0.36$, and $3.90 \pm 0.40 \mathrm{~mm}$ at baseline, and changed to $3.18 \pm 0.42,3.74 \pm 0.36$, and $4.10 \pm 0.38 \mathrm{~mm}$, respectively, after a month from PRP completion (Table 3 ). The pupil sizes of all participants were increased after the laser treatments (Table 3). The pupil size increased significantly in both groups in all illumination conditions (Table 3).

\section{Pain responses}

PS was $5.2 \pm 3.0$ for Group 1 (averages of each session PS) and $1.7 \pm 1.4$ for Group 2. There was a significant difference between groups in pain response $(P=0.001)$. 


\section{Discussion}

It is well known that there are some adverse effects of PRP for diabetic retinopathy such as moderate visual loss, macular edema, glare and light sensitivity, headache, some diminished visual field, reduced color vision, nyctalopia, and reduced contrast sensitivity. ${ }^{9,10}$ Choroidal thickness and choroidal blood flow significantly reduces after PRP. ${ }^{11}$ Ocular pain and headache are other problems of PRP. ${ }^{12}$

There are also some adverse effects of PRP on pupil. ${ }^{8,11}$ Patel et $a l^{8}$ presented four cases in which the pupil remained dilated for 6-18 months following panretinal

Table 1 Participants' demographic characteristics

\begin{tabular}{|c|c|c|c|}
\hline & Group 1 & Group 2 & P-value \\
\hline \multicolumn{4}{|l|}{ Age } \\
\hline Mean \pm SD & $62.5 \pm 3.3$ & $63.7 \pm 3.2$ & \multirow[t]{2}{*}{0.728} \\
\hline Range & 56-69 & 58-68 & \\
\hline \multicolumn{4}{|l|}{$B C V A$ (decimal) } \\
\hline Mean \pm SD & $0.42 \pm 0.22$ & $0.46 \pm 0.20$ & \multirow[t]{2}{*}{0.624} \\
\hline Range & $0.01-0.8$ & $0.1-0.8$ & \\
\hline \multicolumn{4}{|c|}{ Axial length (mm) } \\
\hline Mean \pm SD & $23.14 \pm 0.46$ & $23.32 \pm 0.38$ & \multirow[t]{2}{*}{0.834} \\
\hline Range & $21.22-24.20$ & $22.06-24.12$ & \\
\hline \multicolumn{4}{|l|}{$\mathrm{IOP}(\mathrm{mmHg})$} \\
\hline Mean \pm SD & $15.05 \pm 1.32$ & $15.12 \pm 1.15$ & \multirow[t]{2}{*}{0.960} \\
\hline Range & 12-17 & 13-16 & \\
\hline \multicolumn{4}{|l|}{$C M T(\mu m)$} \\
\hline Mean \pm SD & $365 \pm 56$ & $353 \pm 68$ & \multirow[t]{2}{*}{0.512} \\
\hline Range & $226-488$ & $218-492$ & \\
\hline \multicolumn{4}{|l|}{$S C T(\mu m)$} \\
\hline Mean \pm SD & $305 \pm 37$ & $295 \pm 48$ & \multirow[t]{2}{*}{0.672} \\
\hline Range & $202-472$ & $198-434$ & \\
\hline \multicolumn{4}{|l|}{$H b A 1 c$} \\
\hline Mean \pm SD & $9.2 \pm 0.8$ & $9.4 \pm 0.6$ & \multirow[t]{2}{*}{0.866} \\
\hline Range & 7.5-11 & 7.6-10.8 & \\
\hline
\end{tabular}

Abbreviations: BCVA, best-corrected visual acuity; CMT, central macular thickness; IOP, intraocular pressure; SCT, subfoveal choroidal thickness. laser treatment. They reported that those patients complained of difficulties of their vision, especially on sunny days. ${ }^{8}$ Lobes and Bourgon ${ }^{11}$ presented post-laser pupillary abnormalities in his 22 patients' case series. They reported that all patients developed sector palsies of the iris sphincter, whereas 15 patients demonstrated supersensitivity to $0.125 \%$ pilocarpine, four patients developed accommodative paresis, and six patients showed light near dissociation. ${ }^{11}$ There is not sufficient data in literature on pupillary changes after retinal laser treatments. However, in the present study, we have proved via automated infrared pupillary measurements that pupil sizes were significantly increased after PRP.

Pascal has some advantages over conventional laser such as lower levels of pain-anxiety-headache and short exposure time. ${ }^{13-15}$ Al-Hussainy et al ${ }^{14}$ reported that shortening exposure time of retinal laser is significantly less painful, but equally effective as conventional parameters. Similarly, in our study, the PS was significantly lower in PASCAL laser group. However, the pupil was affected from PRP for both laser systems.

Previous studies showed that the therapeutic destruction of isolated retinal areas with lasers is accompanied by the unavoidable destruction of normal adjacent retinal tissue. $^{5,6}$ Schiodte ${ }^{16}$ have suggested that lasers damage short ciliary nerves as they traverse suprachoroidal space, nerves that left the ciliary ganglion while moving to the ciliary body and iris. We have not seen any direct damage to the iris in any of the participants, so we hypothesize that increased pupil size in both group of patients receiving PRP maybe secondary to damage to short posterior ciliary nerves. The pupil size was more affected in conventional laser group than Pascal group. We think that intense laser burns via conventional laser may cause more damage on ciliary nerves than less intense laser burn via PASCAL. Sheth et al ${ }^{17}$ documented that the total energy of the PASCAL system is a third of that of conventional lasers. Kaufman ${ }^{18}$ worked on monkeys and reported that the severity of morphological changes in nerves passing through the choroid depended directly on the intensity of the laser burn.

Table 2 Parameters of panretinal laser treatments in both groups

\begin{tabular}{|c|c|c|c|c|c|c|}
\hline Power $(m W)$ & $\begin{array}{l}\text { Spot size } \\
\quad(\mu \mathrm{m})\end{array}$ & Spot count (total) & $\begin{array}{l}\text { Duration } \\
\text { (ms) }\end{array}$ & Pattern & $\begin{array}{l}\text { Session count } \\
\quad(\text { total })\end{array}$ & $\begin{array}{l}\text { Laser treatment duration (total) } \\
\text { (min) }\end{array}$ \\
\hline \multicolumn{7}{|l|}{ Group 1} \\
\hline $\begin{array}{l}242 \pm 42(\text { Mean } \pm \text { SD) } \\
200-500 \text { (Range) }\end{array}$ & 400 & $\begin{array}{c}1812 \pm 142(\text { Mean } \pm S D) \\
1420-1938 \text { (Range) }\end{array}$ & $200-400$ & Single spot & $3-4$ & $\begin{array}{c}4.68 \pm 0.60(\text { Mean } \pm S D) \\
4.20-6.42 \text { (Range) }\end{array}$ \\
\hline \multicolumn{7}{|l|}{ Group 2} \\
\hline $\begin{array}{l}528 \pm 68(\text { Mean } \pm \text { SD) } \\
350-900 \text { (Range) }\end{array}$ & 400 & $\begin{array}{c}2018 \pm 132(\text { Mean } \pm S D) \\
1624-2208 \text { (Range) }\end{array}$ & $20-30$ & $3 \times 3$ & 1 & $\begin{array}{c}1.48 \pm 0.30(\text { Mean } \pm S D) \\
1.18-1.82(\text { Range })\end{array}$ \\
\hline
\end{tabular}

\footnotetext{
${ }^{a}$ The intended spot size on the retina (after contact lens magnification).
} 
Table 3 Pupillary measurements of participants (mm)

\begin{tabular}{lccc}
\hline & Photopic & Mesopic & Scotopic \\
\hline Group 1 & & \\
$\quad$ Before & & & \\
$\quad$ Mean \pm SD & $2.88 \pm 0.34$ & $3.38 \pm 0.40$ & $3.95 \pm 0.38$ \\
$\quad$ Range & $2.25-3.12$ & $2.59-4.36$ & $2.98-5.02$ \\
After & & & \\
$\quad$ Mean \pm SD & $3.64 \pm 0.42$ & $4.18 \pm 0.42$ & $4.58 \pm 0.48$ \\
$\quad$ Range & $2.96-4.35$ & $3.35-5.58$ & $3.66-6.22$ \\
P-value & $<0.001^{\mathrm{a}}$ & $<0.001^{\mathrm{a}}$ & $<0.001^{\mathrm{a}}$ \\
Group 2 & & & \\
Before & & & \\
$\quad$ Mean \pm SD & $2.90 \pm 0.38$ & $3.43 \pm 0.36$ & $3.90 \pm 0.40$ \\
$\quad$ Range & $2.54-3.46$ & $2.82-4.32$ & $2.92-4.98$ \\
After & & & \\
$\quad$ Mean $\pm S D$ & $3.18 \pm 0.42$ & $3.74 \pm 0.36$ & $4.10 \pm 0.38$ \\
$\quad$ Range & $2.68-3.98$ & $3.02-4.62$ & $3.14-5.38$ \\
P-value & $0.018^{\mathrm{a}}$ & $0.014^{\mathrm{a}}$ & $0.014^{\mathrm{a}}$ \\
\hline
\end{tabular}

${ }^{a}$ Significant difference ( $P$-values from paired sample $t$-test).

Pupillary constriction is a part of the near reflex with accommodation and convergence. Although occurring in association with accommodation and convergence, it does not depend on either one. ${ }^{19}$ Increased pupil size may cause difficulties on near vision of the patients. Also highorder aberrations maybe increased and photophobia may occur. Physicians may need different type of laser or better delivery systems to avoid pupil size changes after PRP. It may be advisable to use less laser spots and power as possible for only the carefully selected ischemic peripheral retina. Maybe, physicians should use fewer lasers and more intravitreal anti-VEGFs. An interesting previous study on patients with proliferative diabetic retinopathy showed that treatment with only ranibizumab resulted in visual acuity that was noninferior to PRP treatment at 2 years. ${ }^{20}$

This study has posed several limitations. Pupillary changes after PRP via other new laser systems (such as navigated-laser system) was not studied. The strongest side of the study is being the first study that reports objective pupillary measurements after PRP treatments via two different laser systems.

In sum, we have demonstrated via Sirius-automated infrared pupillary measurements that PRP via conventional laser and PASCAL may significantly influence pupil size. Although PASCAL causes less damage on normal tissues, we may still need safer type of laser device protocol to avoid pupillary changes after PRP. Further studies are thus needed to see possible pupillary changes after other new laser systems. Also possible visual effects of increased pupil size after PRP should be studied.

\section{Summary}

What was known before

- We did not know that panretinal laser treatments affect pupil size or not.

What this study adds

- We have demonstrated first time via Sirius-automated infrared pupillary measurements that panretinal laser photocoagulation via conventional laser and PASCAL may significantly increase pupil size.

- Although PASCAL causes less damage on normal tissues, we may still need safer laser devices and procedures to avoid pupillary changes after laser treatments.

\section{Conflict of interest}

The authors declare no conflict of interest.

\section{References}

1 Meyer-Schwickerath G. Light coagulation; a method for treatment and prevention of the retinal detachment. Albrecht Von Graefes Arch Ophthalmol 1954; 156: 2-34.

2 The Diabetic Retinopathy Study Research Group. Indications for photocoagulation treatment of diabetic retinopathy. Diabetic Retinopathy Study report number 14 Int Ophthalmol Clin 1987; 27: 239-253.

3 Early Treatment Diabetic Retinopathy Study Research Group. Early photocoagulation for diabetic retinopathy. ETDRS report number 9. Ophthalmology 1991; 98: 766-785.

4 British Multicenter Study Group. Photocoagulation for proliferative diabetic retinopathy: a randomized controlled clinical trial using the xenon-arc. Diabetologia 1984; 26: 109-115.

5 Rosner M, Solberg Y, Turetz J, Belkin M. Neuroprotective therapy for argon-laser induced retinal injury. Exp Eye Res 1997; 65: 485-495.

6 Solberg Y, Dubinski G, Tchirkov M, Belkin M, Rosner M. Methylprednisolone therapy for retinal laser injury. Surv Ophthalmol 1999; 44(Suppl 1): S85-S92.

7 Ebrahim B, Frohman L, Zarbin M, Bhagat N. Tonic pupil following pars plana vitrectomy and endolaser. Case Rep Med 2009; 2009: 970502.

8 Patel JI, Jenkins L, Benjamin L, Webber S. Dilated pupils and loss of accommodation following diode panretinal photocoagulation with sub-tenon local anaesthetic in four cases. Eye (Lond) 2002; 16(5): 628-632.

9 Fong DS, Girach A, Boney A. Visual side effects of successful scatter laser photocoagulation surgery for proliferative diabetic retinopathy: a literature review. Retina 2007; 27(7): 816-824.

10 Dowler JG. Laser management of diabetic retinopathy. J $R$ Soc Med 2003; 96(6): 277-279.

11 Lobes Jr LA, Bourgon P. Pupillary abnormalities induced by argon laser photocoagulation. Ophthalmology 1985; 92(2): 234-236.

12 Zakrzewski PA, O'Donnell HL, Lam WC. Oral versus topical diclofenac for pain prevention during 
panretinalphotocoagulation. Ophthalmology 2009; 116(6): 1168-1174.

13 Salman AG. Pascal laser versus conventional laser for treatment of diabetic retinopathy. Saudi J Ophthalmol 2011; 25(2): 175-179.

14 Al-Hussainy S, Dodson PM, Gibson JM. Pain response and follow-up of patients undergoing panretinal laser photocoagulation with reduced exposure times. Eye 2008; 22 96-99.

15 Nagpal M, Marlecha S, Nagpal K. Comparison of laser photocoagulation for diabetic retinopathy using 532-nm standard laser versus multispot pattern scan laser. Retina 2010; 30: 452-458.

16 Schiodte SN. Effects of choroidal nerves after panretinal xenon arc and argon laser photocoagulation. Acta Ophthalmol (Copenh) 1984; 62: 244-255.
17 Sheth S, Lanzetta P, Veritti D, Zucchiatti I, Savorgnani C, Bandello F. Experience with the Pascal photocoagulator: an analysis of over 1,200 laser procedures with regard to parameter refinement. Indian J Ophthalmol 2011; 59: 87-91.

18 Kaufman PL. Parasympathetic denervation of the ciliary muscle following retinal photocoagulation. Trans Am Ophthalmol Soc 1990; 88: 513-553.

19 Thompson HS. The pupil. In: Moses RA (ed). Adler's Physiology of the Eye, 7th ed. Mosby-Year Book: St Louis, MO, USA, 1982, pp 326.

20 Gross JG, Glassman AR, Jampol LM, Inusah S, Aiello LP, Antoszyk AN et al. Panretinal photocoagulation vs intravitreous ranibizumab for proliferative diabetic retinopathy: a randomized clinical trial. JAMA 2015; 314 (20): 2137-2146. 